\title{
A green and simple method for preparation of an efficient palladium adsorbent based on cysteine functionalized 2,3-dialdehyde cellulose
}

\author{
Changqing Ruan • Maria Strømme • \\ Jonas Lindh
}

Received: 8 April 2016/ Accepted: 27 May 2016/Published online: 6 June 2016

(C) Springer Science+Business Media Dordrecht 2016

\begin{abstract}
A green and efficient adsorbent for adsorption of palladium ions was prepared from 2,3-dialdehyde cellulose (DAC) originating from nanocellulose from the green algae Cladophora. The DAC was functionalized with cysteine via reductive amination in a convenient one-pot procedure to provide the adsorbent. The adsorption properties for adsorbing palladium(II) ions, including capacity, adsorption isotherm and kinetics, were studied. The successful reductive amination of cysteine with 2,3-dialdehyde cellulose was confirmed by FT-IR, elemental analysis and XPS. The adsorbent was characterized by SEM, XRD, gas adsorption and TGA. The adsorbent had a high adsorption capacity (130 mg palladium per gram adsorbent) and enabled fast adsorption of palladium(II) ions from solution (80\% of maximum capacity reached in $2 \mathrm{~h}$ ). Adsorbent materials suitable for both filters (fibrous) and column matrixes (spherical particles) could be obtained in an efficient manner by controlling the degree of oxidation while producing the DAC material.
\end{abstract}

Keywords 2,3-dialdehyde cellulose $\cdot$ Palladium adsorption $\cdot$ Cellulose beads $\cdot$ Nanocellulose

C. Ruan · M. Strømme · J. Lindh $(\square)$

Nanotechnology and Functional Materials, Department of Engineering Sciences, Uppsala University, Box 534,

75121 Uppsala, Sweden

e-mail: jonas.lindh@angstrom.uu.se

\section{Introduction}

The transition metal palladium is widely used in e.g. electronic-, pharmaceutical- and chemical industry, due to its specific physical and chemical properties. The recovery and extraction of palladium from industrial waste has gained considerable interest due to high demand, low natural abundance (Barakat et al. 2006; Liu et al. 2003) and the heavy-metal toxic effect palladium exerts to animals, plants and humans (Nolan and Lippard 2008). Furthermore, palladium is a frequently used catalyst for the formation of $\mathrm{C}-\mathrm{C}$ bonds in the production of pharmaceuticals and is allowed only in sub 5 ppm amounts in pharmaceuticals (Products CfPM 2002). Therefore, searching for efficient methods for palladium recovery and extraction is important. Further, palladium can be used as a benchmark transition metal and knowledge gained about palladium recovery could likely be useful for developing recovery methods also for other transition metals, e.g. platinum, rhodium and ruthenium.

Some well-known methods including chemical precipitation (Vincent et al. 2007), ion exchange (Anpilogova et al. 2014), solvent extraction (Paiva et al. 2014), membrane separation (Li et al. 1994) and adsorption (Gupta et al. 2003; Sharma and Rajesh 2014) are employed to recover palladium. Among the above-mentioned methods, adsorption is highly efficient and is frequently used. Several studies have investigated palladium ions adsorption using e.g. thiol- 
functionalized silica (Lim et al. 2011; Qishu et al. 2012), modified chitin or chitosan (Fujiwara et al. 2007; Wasikiewicz et al. 2007) and functionalized polymers or resins (Awual et al. 2013; JermakowiczBartkowiak et al. 2005; Venkatesan et al. 2005). Among the adsorbents, some are not environmentally benign and rely on a petrol feedstock, whereas others are green, e.g. chitin or chitosan, but suffer from lower adsorption capacity. Furthermore, the ease of extracting or desorbing the palladium ions from the adsorbent in order to recycle the precious metal greatly varies between the different materials, where the biopolymers can be simply incinerated at a relatively low temperature to obtain the pure palladium, a procedure that is not convenient for e.g. the silica-based adsorbents.

Several aspects are important to consider when developing an efficient material for adsorption. Besides the obvious advantages of having a large surface area, ample coordination sites, and efficient coordination of the adsorbate, the practical execution of the adsorption is a key aspect e.g. by using filters or columns. For filters, a material with fibrous character is desired as it is beneficial for the strength of filters. A material used as matrix in columns on the other hand should preferably have a spherical morphology to keep backpressure to a minimum.

Cellulose is the most abundant renewable biopolymer in nature and is a promising raw material in terms of cost of production and ease of modification (Klemm et al. 2005). Periodate oxidation of cellulose is a highly specific reaction for converting the 2,3-dihydroxyl groups to 2,3-dialdehyde groups without significant side reactions (Bruneel and Schacht 1993; Maekawa and Koshijima 1984). The resulting aldehyde groups can be further transformed to e.g. carboxyl groups (Maekawa and Koshijima 1984; Takaichi et al. 2014), primary alcohols (Casu et al. 1985), or imines (Schiff bases) with primary amines (Sabzalian et al. 2014), which enables the use of 2,3-dialdehyde cellulose (DAC) as a suitable starting material in a wide range of applications e.g. as an adsorbent of heavy metals (Nada and Hassan 2006), DNA (Su et al. 2007) or dyes (Kitkulnumchai et al. 2008).

Our group has made research efforts towards finding useful applications of Cladophora nanocellulose (Carlsson et al. 2014; 2015; Metreveli et al. 2014; Nyholm et al. 2011; Razaq et al. 2012). The use of Cladophora nanocellulose is well in line with the concept of sustainable industrial scale processing, as the source of this cellulose is abundant and, in addition, Cladophora algae are typically considered undesirable pollutants, known for forming large, foulsmelling, algal mats and having adverse effects on the aquatic ecosystem as well as harbouring bacterial pathogens (Mihranyan 2011). Furthermore, no large scale applications for this source of cellulose exists so the use of this source is not in conflict with other applications e.g. food production, fuel production or building materials. Recently, we reported a new and convenient method for producing DAC beads by periodate oxidation of Cladophora nanocellulose (Lindh et al. 2014; 2016). The method provides spontaneous bead formation in a one-pot procedure under aqueous conditions at high degrees of oxidation, circumventing the traditional tedious procedure for bead formation typically involving: (1) dissolution of cellulose, (2) shaping of the beads and (3) regeneration of the polysaccharide, where one or more steps typically employ environmentally harmful solvents (Gericke et al. 2013). The periodate oxidation can also be controlled to produce materials with fibrous character by performing less extensive periodate oxidation. Based on our work on periodate oxidation of Cladophora cellulose we now introduce a green, renewable and efficient material for adsorption of palladium ions. Different kinds of DAC was prepared by periodate oxidation and were further functionalized via reductive amination with cysteine in a facile onepot procedure. The obtained materials were used for palladium ion adsorption and were characterized by SEM, XRD, gas adsorption, FTIR, TGA and XPS.

\section{Experimental}

\section{Materials}

Nanocellulose from Cladophora algae was provided by FMC Biopolymer. Sodium metaperiodate $\left(\mathrm{NaIO}_{4}\right)$, hydroxylamine hydrochloride $\left(\mathrm{NH}_{2} \mathrm{OH} \cdot \mathrm{HCl}\right), 2$-picoline-borane $\left(\mathrm{C}_{6} \mathrm{H}_{7} \cdot \mathrm{BH}_{3}\right)$, L-cysteine and other chemicals used were of analytical or reagent grade and were used as received. Deionized water was used throughout the experimental procedures.

\section{Preparation of DAC}

The preparation of DAC proceeded according to literature procedure (Lindh et al. 2014). In short, 
Cladophora nanocellulose, $20 \mathrm{~g}$ in $1.5 \mathrm{~L}$ of water was mixed with $134 \mathrm{~g}$ of sodium metaperiodate ( $5 \mathrm{~mol}$ per mol of anhydroglucose units) dissolved in the same medium as the cellulose. The periodate-containing reaction mixture was carefully wrapped in aluminum foil to avoid light exposure. The reaction mixture was vigorously stirred at room temperature in the dark for 13 days. Aliquots were withdrawn after 2 and 13 days (750 mL each time) and named 2D-DAC and 13DDAC, respectively. The withdrawn aliquots were immediately quenched via the addition of ethylene glycol and washed repeatedly with water to provide pure DAC. DAC samples were dried under reduced pressure at $40{ }^{\circ} \mathrm{C}$ and used in their dry state for further reactions.

\section{Reductive amination of DAC}

Dried DAC (2D-DAC or 13D-DAC), $1 \mathrm{~g}$, was added to a $250 \mathrm{~mL}$ round-bottom flask, which contained $100 \mathrm{~mL}$ methanol. After vigorous stirring, to make the DAC suspend well, L-cysteine (7.57 g, 10 equiv.) and 2-picoline-borane (2.67 g, 4 equiv.) were added successively. The reaction mixture was stirred at room temperature for $24 \mathrm{~h}$. The products, named 2DDAC-LC and 13D-DAC-LC, were washed with distilled water followed by ethanol and dried under reduced pressure at $40{ }^{\circ} \mathrm{C}$ for further use.

\section{Scanning electron microscopy}

Scanning electron micrographs were taken with a LEO1550 field-emission SEM instrument (Zeiss, Germany) operated at $1 \mathrm{kV}$ with an in-lens secondary electron detector. Samples were mounted on aluminum stubs by means of double-sided adhesive carbon tape and sputtered with gold/palladium to avoid charging effects.

\section{X-ray diffraction}

$\mathrm{X}$-ray diffraction (XRD) analysis was performed with a diffractometer having Bragg-Bretano geometry $(\mathrm{Cu}$ $\mathrm{K} \alpha$ radiation; $\lambda=1.54 \AA$ ) (D5000, Siemens/Bruker, Germany). The crystallinity index of the nanocellulose and its derivatives was calculated using the Segal empirical equation (Segal et al. 1959).
$C r I=\frac{I_{22.5^{\circ}}-I_{18^{\circ}}}{I_{22.5^{\circ}}}$

where $\mathrm{CrI}$ stands for crystallinity index of cellulose. $I_{22.5^{\circ}}$ represents the peak diffraction intensity corresponding to crystalline cellulose and $I_{18^{\circ}}$ is the peak diffraction intensity corresponding to the amorphous sections at 2 -Theta, $22.5^{\circ}$ and $18^{\circ}$, respectively.

Nitrogen sorption isotherm analysis

Nitrogen gas adsorption and desorption isotherms of Cladophora nanocellulose and its derivatives were performed on an ASAP 2020 instrument (Micromeritics, USA). The specific surface area was calculated based on the BET (Brunauer et al. 1938) method during adsorption with the ASAP 2020 software, while the pore size distribution was determined according to the density functional theory (DFT) method using the model for nitrogen at $77 \mathrm{~K}$.

Fourier transform infrared spectroscopy (FTIR)

FTIR spectra were recorded on a Tensor 27 FTIR spectrometer (Bruker, Germany). The resolution was set to $4 \mathrm{~cm}^{-1}$ with 64 scans over a range of $4000-400 \mathrm{~cm}^{-1}$. Samples were ground with $\mathrm{KBr}$ and prepared into pellets for FTIR measurements.

Thermal gravimetric analysis (TGA)

Samples were analyzed with a TGA/SDTA851 instrument (Mettler Toledo, Switzerland), at a temperature range of $25-800{ }^{\circ} \mathrm{C}$ with a heating rate of $5^{\circ} \mathrm{C}$ per minute under air or nitrogen atmosphere.

X-ray photoelectron spectroscopy (XPS)

High-resolution XPS spectra for C, N, O, S, and Pd were performed with an ESCA instrument (Physical Electronics, U.S.) equipped with an $\mathrm{Al} \mathrm{K} \alpha$ source $(1486.6 \mathrm{eV}, 45 \mathrm{~W})$. The analyzer was operated at $23.5 \mathrm{eV}$ pass energy, while electron and $\mathrm{Ar}^{+}$gun neutralization was applied to compensate for surface charging effects. Elemental atomic concentrations and ratios were calculated from the XPS peak areas with the Multipak software (Physical Electronics, U.S.). 
Determination of aldehyde content

In order to determine the degree of oxidation (D.O.), elemental analysis was conducted. The DAC samples were transformed to aldoximes via Schiff base reactions with hydroxylamine according to a literature procedure (Lindh et al. 2014) and were analyzed for elemental composition ( $\mathrm{C}, \mathrm{H}$, and $\mathrm{N})$. To a stirred $100 \mathrm{~mL}$ RB-flask was added dried DAC (100 mg), $40 \mathrm{~mL}$ of acetate buffer ( $\mathrm{pH} 4.5$ ), and $1.65 \mathrm{~mL}$ of hydroxylamine solution (aqueous, $50 \mathrm{wt} \%$ ). The reaction mixture was stirred at room temperature for $24 \mathrm{~h}$. The product was thoroughly washed with water and dried under reduced pressure prior to elemental analysis. The term "degree of oxidation" (D.O.) represents the ratio of 2,3-alcohols in the anhydroglucose units that has been transformed into their corresponding aldehydes. The highest degree of oxidation, i.e. $100 \%$, corresponds to all anhydroglucose units being converted to the corresponding noncyclic 2,3-dialdehyde structures, which would correspond to approximately $12.5 \mathrm{mmol}$ of aldehyde groups per gram of cellulose.

\section{Determination of cysteine content}

The degree of functionalization, i.e. the degree of cysteine coupled to the DAC, was determined via elemental analysis $(\mathrm{C}, \mathrm{H}, \mathrm{N}$ and $\mathrm{S})$ of the 2D-DAC-LC and 13D-DAC-LC samples. The term "degree of functionalization" represents the ratio of the original 2,3-alcohols in the anhydroglucose units that has been functionalized with L-cysteine via reductive amination of the periodate induced 2,3-dialdehydes. The highest degree of functionalization, i.e. $100 \%$, corresponds to all of the original 2,3-dialcohols being functionalized with L-cysteine, which would correspond to approximately $12.5 \mathrm{mmol}$ of cysteine per gram of cellulose.

\section{Adsorption experiments}

A series of $\mathrm{Pd}(\mathrm{OAc})_{2}$ solutions of various initial concentrations $\left(10-600 \mathrm{mg} / \mathrm{L}\right.$ of $\left.\mathrm{Pd}(\mathrm{OAc})_{2}\right)$ were prepared by dissolving given amounts of $\mathrm{Pd}(\mathrm{OAc})_{2}$ in acetonitrile. $10 \mathrm{~mL}$ aliquots were placed in $15 \mathrm{~mL}$ plastic vials and a given amount of adsorbent was added to each vial. Each vial was then sealed and fixed on a tube rotator (VWR Tube Rotator) and rotated for $24 \mathrm{~h}$. The vials were centrifuged at $4700 \mathrm{~g}$ for $10 \mathrm{~min}$.
An aliquot of the supernatant was then withdrawn and analyzed by UV-vis spectroscopy (Shimadzu, UV1800, Japan). Blank solutions containing equivalent initial concentration of Pd but without addition of adsorbent were subjected to the same procedure. Adsorption isotherms were carried out at room temperature, $22.5^{\circ} \mathrm{C}$.

The adsorption isotherm data were analyzed by both the Langmuir (2) and Freundlich (3) models, which are expressed in linear form as (Freundlich 1906; Langmuir 1918),

$\frac{C_{e}}{q_{e}}=\frac{1}{q_{0} b}+\frac{1}{q_{0}} C_{e}$

$\ln q_{e}=\ln K_{F}+\frac{1}{n} \ln C_{e}$

respectively. Adsorption kinetics for palladium ion adsorption on DAC-based adsorbent was explored in a batch experiment. A number of vials $(15 \mathrm{~mL})$ containing the same concentration of $\mathrm{Pd}(\mathrm{OAc})_{2}(100 \mathrm{mg} /$ L) with the same amount of adsorbent $(4 \mathrm{mg})$ were prepared, and after 2, 4, 8, 15, 30, 60, 120, 240, 360, $480,1440,1860$ and $4320 \mathrm{~min}$, the suspension in the corresponding vial was filtered through a PTFE (Polytetrafluoroethylene) filter. Adsorption amount was calculated by the difference of the concentration of initial solution of $\mathrm{Pd}(\mathrm{OAc})_{2}$ and the filtrate, both of which were measured with UV-vis spectrophotometer. Blank controls were taken without addition of adsorbent. All the experiments were performed at room temperature $\left(22.5^{\circ} \mathrm{C}\right)$.

\section{Results and discussion}

In this study two types of DACs were prepared, differing in the degree of oxidation (D.O.). One type was oxidized to $30 \%$ D.O. by carrying out the oxidation for two days (2D-DAC) and the other had a D.O. of $85 \%$, which was achieved by performing the oxidation for 13 days (13D-DAC). The 2D-DAC and the 13D-DAC were subjected to reductive amination with L-cysteine using 2-picoline-borane as reducing agent, providing 2D-DAC-LC with a degree of Lcysteine functionalization of $9 \%$ and 13D-DAC-LC with a degree of L-cysteine functionalization of $49 \%$ (Fig. 1). 2-picoline-borane is a non-toxic and efficient reducing agent, which is selective towards imines and 


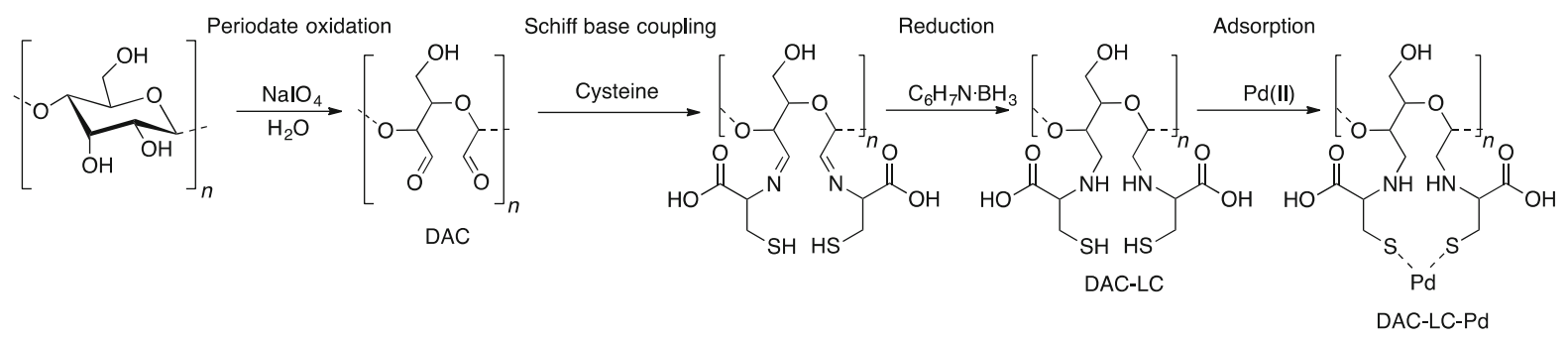

Fig. 1 Reaction scheme for the preparation of cysteine functionalized DAC and a plausible coordination mode for the adsorption of $\operatorname{Pd}(\mathrm{II})$ ions

thus allows the direct conversion of carbonyl compounds into amines in a one-pot procedure (Cosenza et al. 2011; Ruhaak et al. 2010; Sato et al. 2004). The functionalized DACs contain plentiful coordination sites, which enable efficient adsorption of palladium. The L-cysteine functionalized DAC materials were then subjected to the adsorption of Pd(II) ions and provided high adsorption capacity. For convenience, the absorbents 2D-DAC-LC and 13D-DAC-LC loaded with palladium were named 2D-DAC-LC-Pd and 13D-DAC-LC-Pd, respectively.

Adsorption isotherm and kinetics

Equilibrium adsorption time is used to evaluate the effect of the contact time between adsorbent and adsorbate (Awual et al. 2013). The results of the relationship between adsorption amount and contact time are shown in Fig. 2a. The data indicate that the adsorption of $\mathrm{Pd}(\mathrm{II})$ ions was fast and $80 \%$ of maximum adsorption amount could be reached after $2 \mathrm{~h}$. As the adsorption almost reached equilibrium in $24 \mathrm{~h}$, this time was selected in further experimental work for obtaining the adsorption isotherms.

An adsorption isotherm can be used to characterize the relationship between the adsorbent and the adsorbate. This provides a relationship between the concentration of adsorbate in the solution and the amount of adsorbate adsorbed on to solid phase when the two phases are at equilibrium (Sağ 2001). The adsorption isotherms for Pd(II) on 2D-DAC-LC and 13D-DACLC are presented in Fig. 2b. The amount of metal ions adsorbed at equilibrium per unit mass $\left(q_{e}\right)$ of the DACLC increased first with the increase of the equilibrium concentration of solute $\left(C_{e}\right)$ then almost reached a plateau value, which represents saturation of the active chelation sites on DAC-LC.

The Langmuir plot $\left(C_{e} / q_{e}\right.$ against $\left.C_{e}\right)$ was used to obtain Langmuir constants, $q_{o}$ and $b$, related to the adsorption capacity and energy of adsorption,
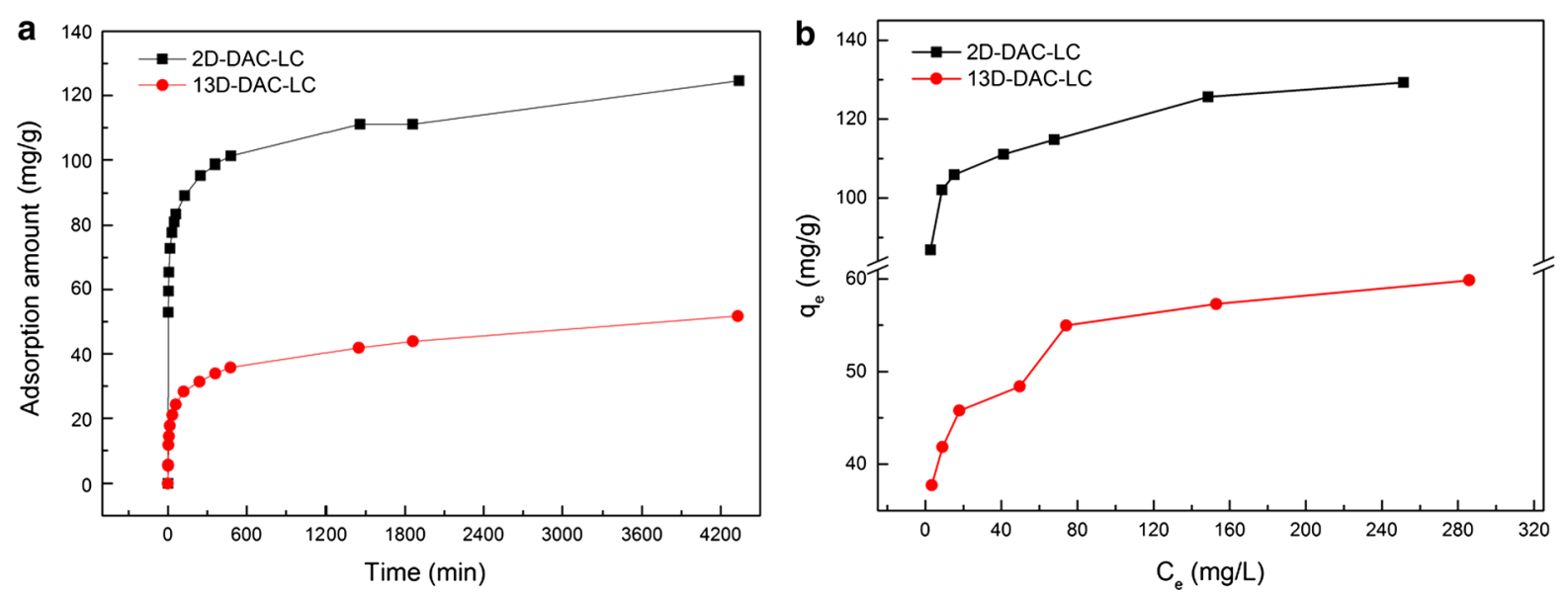

Fig. 2 a Effect of contact time on Pd(II) adsorption and $\mathbf{b}$ adsorption isotherm for Pd(II) on 2D-DAC-LC and 13D-DAC-LC. Connecting line in $\mathbf{a}$ and $\mathbf{b}$ only to serve as guide to the eye 
respectively. From the Freundlich model, the $n$ and $K_{F}$ are provided, which relates to the adsorption intensity and the adsorption capacity, respectively. All the parameters are shown in Table 1. The Langmuir model yielded high correlation coefficient values $\left(\mathrm{R}^{2}\right)$ 0.9988 and 0.9985 and the $q_{o}$ values were $130.4 \mathrm{mg} / \mathrm{g}$ and $60.7 \mathrm{mg} / \mathrm{g}$ for Pd(II) adsorbed on 2D-DAC-LC and 13D-DAC-LC, respectively. Values for adsorption capacity of other adsorbents presented in literature are given in Table 2 for comparison. The somewhat surprising higher adsorption capacity for 2D-DAC-LC compared to 13D-DAC-LC, despite the lower content of coordinating cysteine groups, is probably a result of lower accessible surface area of the bead shaped 13D-DAC-LC (see Table 3).

The thermodynamic parameter, the Gibbs free energy $\left(\Delta G^{\circ}\right)$ (see Table 1), for 2D-DAC-LC and 13D-DAC-LC was evaluated from the Langmuir constant using the equation (Gupta et al. 2006)

$\Delta G^{\circ}=-R T \ln b$

where $b$ is the equilibrium constant and $R$ is the universal gas constant. The negative values of $\Delta \mathrm{G}^{\circ}$ confirm the feasibility of the adsorption process.

In order to investigate the mechanism of adsorption, the kinetic models for pseudo first order (5) (Lagergren 1898) and pseudo second order (6) (Sparks 1986) were applied. The pseudo first order model is expressed by

$\log \left(q_{e}-q_{t}\right)=\log q_{e}-\frac{k_{1} t}{2.303}$

where $q_{e}$ and $q_{t}$ are the amounts of adsorbed $\mathrm{Pd}(\mathrm{II})$ $(\mathrm{mg} / \mathrm{g})$ at equilibrium and time $t$, respectively, and $k_{1}$ $\left(\mathrm{min}^{-1}\right)$ is the rate constant of pseudo-first-order adsorption. The pseudo second order model is given by

$\frac{t}{q_{t}}=\frac{1}{k_{2} q_{e}^{2}}+\frac{t}{q_{e}}$ where $k_{2}[\mathrm{~g} /(\mathrm{mg} \mathrm{min})]$ is the rate constant of pseudosecond-order adsorption, $q_{e}$ is the amount of $\mathrm{Pd}(\mathrm{II})$ adsorbed $(\mathrm{mg} / \mathrm{g})$ at equilibrium, and $q_{t}$ is the amount of the adsorption $(\mathrm{mg} / \mathrm{g})$ at any time $t$.

Based on the results in Fig. 3, it is obvious that the adsorption behavior is best described by the pseudo second order model, providing a $\mathrm{R}^{2}$ value $>0.99$ for both adsorbents, implying that the adsorption kinetics is largely controlled by chemisorption.

Morphological characterization

SEM micrographs of samples of unmodified Cladophora nanocellulose, 2D-DAC, 13D-DAC and the corresponding ligand functionalized derivatives, before and after adsorption of palladium, are shown in Fig. 4. As previously described (Lindh et al. 2014), extensive periodate oxidation, i.e. 13 days oxidation, provided drastic change to the cellulose morphology (producing beads with a relatively smooth surface), while 2 days oxidation induced a more compact cellulose compared to the unmodified Cladophora nanocellulose. Furthermore, the reductive amination with L-cysteine on DAC provided materials with less compact and more porous texture. Adsorption experiments provided little or no change to the derivatives morphology, neither for 2D-DAC nor 13D-DAC.

\section{FTIR spectroscopy}

Figure 5 illustrates the FTIR spectra of unmodified Cladophora nanocellulose and the functionalized derivatives. The signals at 880 (Fig. 5b) and $1734 \mathrm{~cm}^{-1}$ (Fig. 5c) are assigned to the hemiacetal and carbonyl groups, respectively (Rowen et al. 1951; Spedding 1960). When studying the spectra of Cladophora cellulose, 2D-DAC and 13D-DAC in these two regions, the signals increase as the D.O. increases. Furthermore, after L-cysteine-modification,

Table 1 Adsorption parameters of Pd(II) ions on 2D-DAC-LC and 13D-DAC-LC and Gibbs free energy $\left(\Delta \mathrm{G}^{\circ}\right)$ for adsorption

\begin{tabular}{|c|c|c|c|c|c|c|c|}
\hline \multirow[t]{2}{*}{ Adsorbent } & \multicolumn{3}{|l|}{ Langmuir } & \multicolumn{3}{|c|}{ Freundlich } & \multirow[t]{2}{*}{$-\Delta \mathrm{G}^{\circ}(\mathrm{kJ} / \mathrm{mol})$} \\
\hline & $q_{o}(\mathrm{mg} / \mathrm{g})$ & $b \times 10^{4}(\mathrm{~L} / \mathrm{mol})$ & $R^{2}$ & $K_{F}$ & $n$ & $R^{2}$ & \\
\hline 2D-DAC-LC & 130.4 & 3.566 & 0.9988 & 82.63 & 12.17 & 0.9644 & 25.75 \\
\hline 13D-DAC-LC & 60.7 & 2.496 & 0.9985 & 33.20 & 9.34 & 0.9722 & 24.87 \\
\hline
\end{tabular}


Table 2 Comparison of adsorption capacity of different adsorbents

\begin{tabular}{lll}
\hline Adsorbent & $\begin{array}{l}\text { Adsorption } \\
\text { capacity (mg/g) }\end{array}$ & References \\
\hline Cellulose-MBT & 5 & (Sharma and Rajesh 2014) \\
Native cellulose & 1.87 & (Sharma and Rajesh 2014) \\
Radiation cross-linked carboxymethylchitin hydrogels & 2.68 & (Wasikiewicz et al. 2007) \\
Chitosan resin modified with L-lysine & 109.47 & (Fujiwara et al. 2007) \\
Polystyrene-divinylbenzene functionalized with isothiouronium & 20 & (Venkatesan et al. 2005) \\
Carbon black modified with L-Cysteine & 84 & (Panchompoo et al. 2011) \\
Mesoporous silica functionalized with 3-(((5-ethoxybenzenethiol) & 164.2 & (Awual et al. 2013) \\
$\quad$ imino)methyl)-salicylic acid ligand & & This work \\
2D-DAC-LC & 130.4 & This work \\
13D-DAC-LC & 60.7 &
\end{tabular}

Table 3 Pore volume and SSA of cellulose and its derivatives

\begin{tabular}{llr}
\hline & Pore volume $\left(\mathrm{cm}^{3} / \mathrm{g}\right)$ & SSA $\left(\mathrm{m}^{2} / \mathrm{g}\right)$ \\
\hline Cladophora cellulose & 0.6 & 102 \\
2D-DAC & 0.4 & 47 \\
2D-DAC-LC & 1 & 141 \\
13D-DAC & 0.003 & 1 \\
13D-DAC-LC & 0.3 & 47 \\
\hline
\end{tabular}

a more distinct signal at $1734 \mathrm{~cm}^{-1}$ corresponding to carbonyl groups (Spedding 1960) (comparing ii) and iii), or v) and vi)), verified the introduction of L-cysteine on DAC.
X-ray photoelectron spectroscopy (XPS)

The XPS spectra in wide scans of 2D-DAC, 2D-DACLC, 2D-DAC-LC-Pd, 13D-DAC, 13D-DAC-LC and 13D-DAC-LC-Pd are presented in Fig. 6. From the spectra of 2D-DAC and 13D-DAC, the characteristic peaks of $\mathrm{C} 1 \mathrm{~s}(285 \mathrm{eV})$ and $\mathrm{O} 1 \mathrm{~s}(531 \mathrm{eV})$ are shown. For the spectra of 2D-DAC-LC and 13D-DAC-LC, the additional peaks corresponding to $\mathrm{N} 1 \mathrm{~s}(398 \mathrm{eV})$ and $\mathrm{S}$ $2 p(164 \mathrm{eV})$ peaks can be clearly seen and the spectra of 2D-DAC-LC-Pd and 13D-DAC-LC-Pd reveal the further addition of signals for $\mathrm{Pd} 3 \mathrm{~d} 5(335 \mathrm{eV})$ and $\mathrm{Pd}$ $3 \mathrm{~d} 3(340 \mathrm{eV})$ (Moulder et al. 1992).
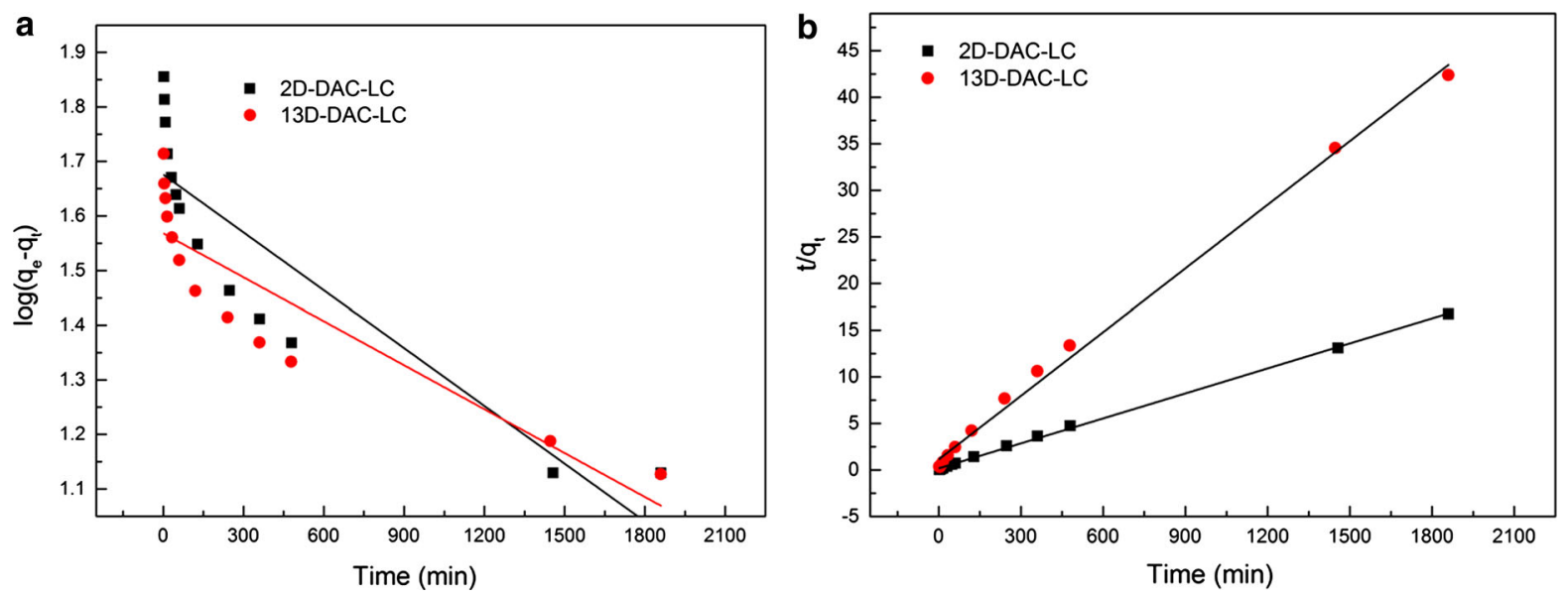

Fig. 3 a Pseudo first order kinetics and b pseudo second order kinetics for Pd(II) on 2D-DAC-LC and 13D-DAC-LC (the concentration of palladium acetate in acetonitrile was $100 \mathrm{mg} / \mathrm{L}$ ) 

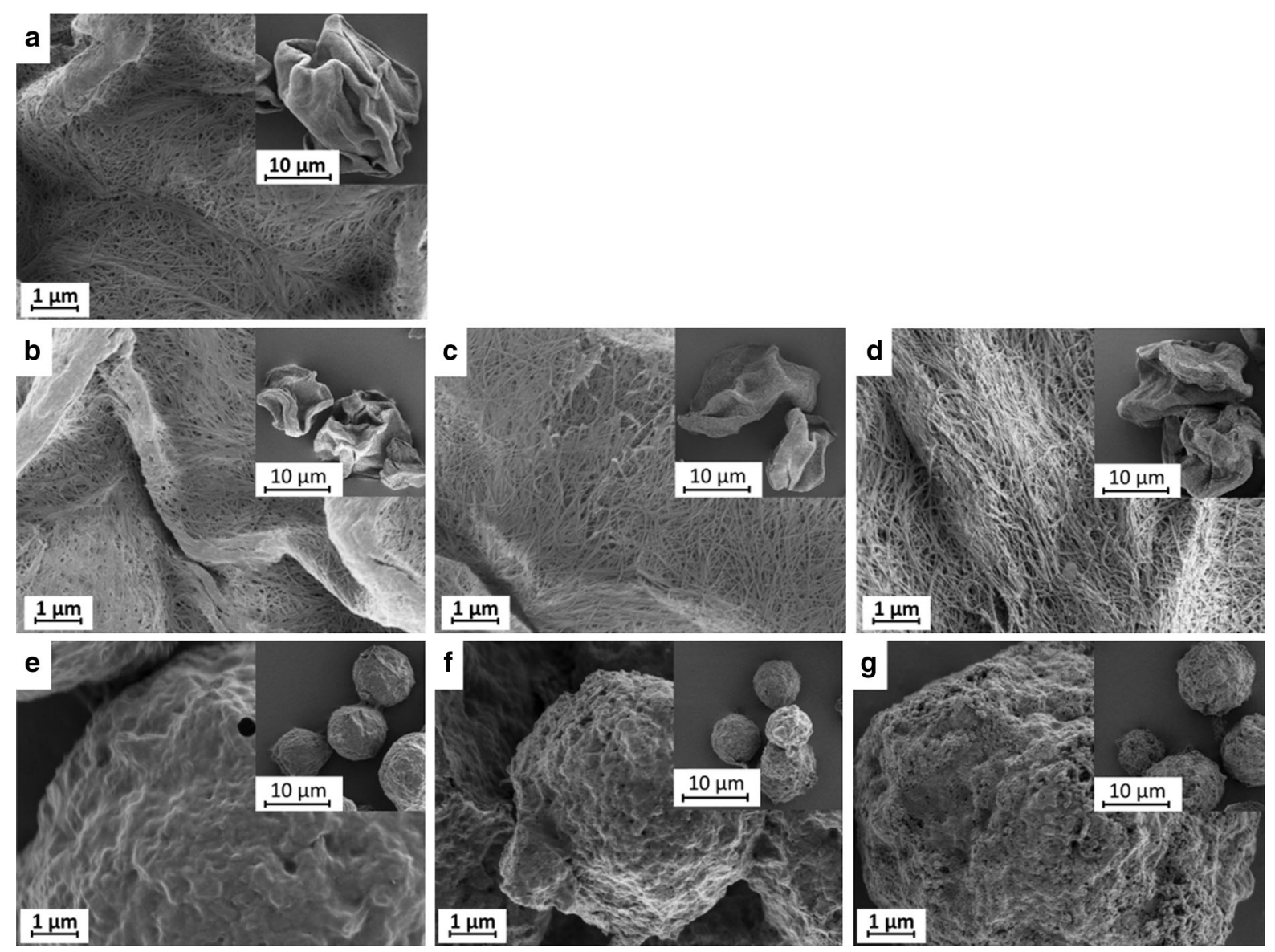

Fig. 4 SEM micrographs of a unmodified Cladophora nanocellulose, b 2D-DAC, c 2D-DAC-LC, d 2D-DAC-LC-Pd, e 13D-DAC, f 13D-DAC-LC and g 13D-DAC-LC-Pd

Thermal gravimetric analysis (TGA)

Thermal stability of nanocellulose, oxidized nanocellulose and the corresponding derivatives was investigated by TGA and the obtained curves are depicted in Fig. 7. It can be seen that degradation of 2D-DAC and 13D-DAC compared to that of unmodified Cladophora nanocellulose is initiated at a lower temperature, both in compressed air and under nitrogen atmosphere. Furthermore, the degradation of 2DDAC-LC and 13D-DAC-LC commences at a lower temperature than that of 2D-DAC and 13D-DAC, which indicates that the thermal stability of materials deriving from nanocellulose decreases after modification in terms of oxidation and L-cysteine modification. On the other hand, final weight percentages of 2DDAC-LC-Pd and 13D-DAC-LC-Pd are substantially higher than for the corresponding material before adsorption of palladium, both in compressed air and nitrogen atmosphere, which confirms the adsorption of palladium ions during the adsorption experiments. These TGA results confirm the XPS results that palladium ions were adsorbed on 2D-DAC-LC and 13D-DAC-LC and also demonstrate that the L-cysteine modification was functional.

Surface area and porosity analysis

Nitrogen sorption isotherms at a temperature of $77 \mathrm{~K}$ and pore size distribution for Cladophora nanocellulose and its derivatives are given in Fig. 8. The pore volume and specific surface area (SSA) of Cladophora nanocellulose and its derivatives are displayed in Table 3. The SSA decreases with increased D.O., two orders of magnitude from 102 to $1 \mathrm{~m}^{2} / \mathrm{g}$ corresponding to unmodified Cladophora nanocellulose and 13D- 

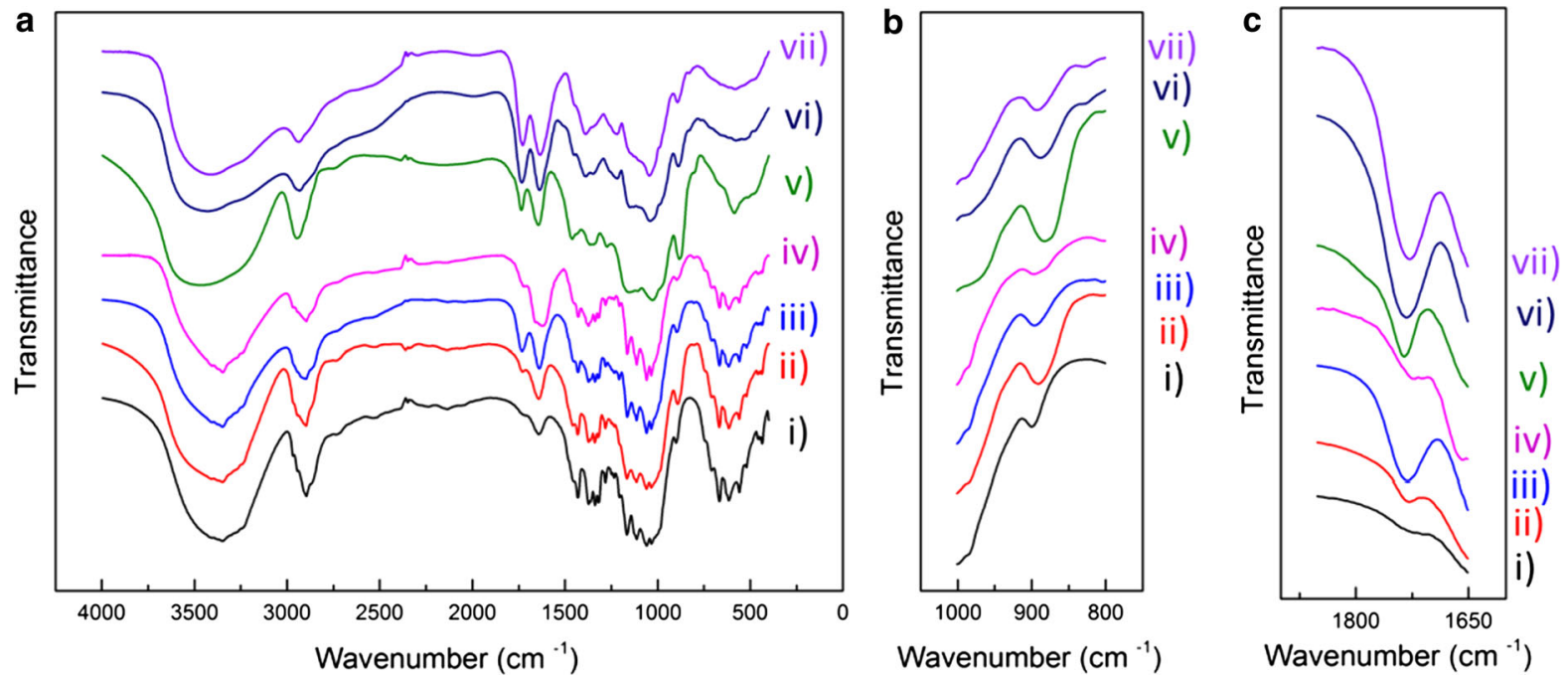

Fig. 5 FT-IR spectra of a 4000-400 $\mathrm{cm}^{-1}$, b $1000-800 \mathrm{~cm}^{-1}$ and c $1850-1650 \mathrm{~cm}^{-1}$ for $i$ ) unmodified Cladophora nanocellulose, ii) 2D-DAC, iii) 2D-DAC-LC, iv) 2D-DAC-LC-Pd, v) 13D-DAC, vi) 13D-DAC-LC and vii) 13D-DAC-LC-Pd
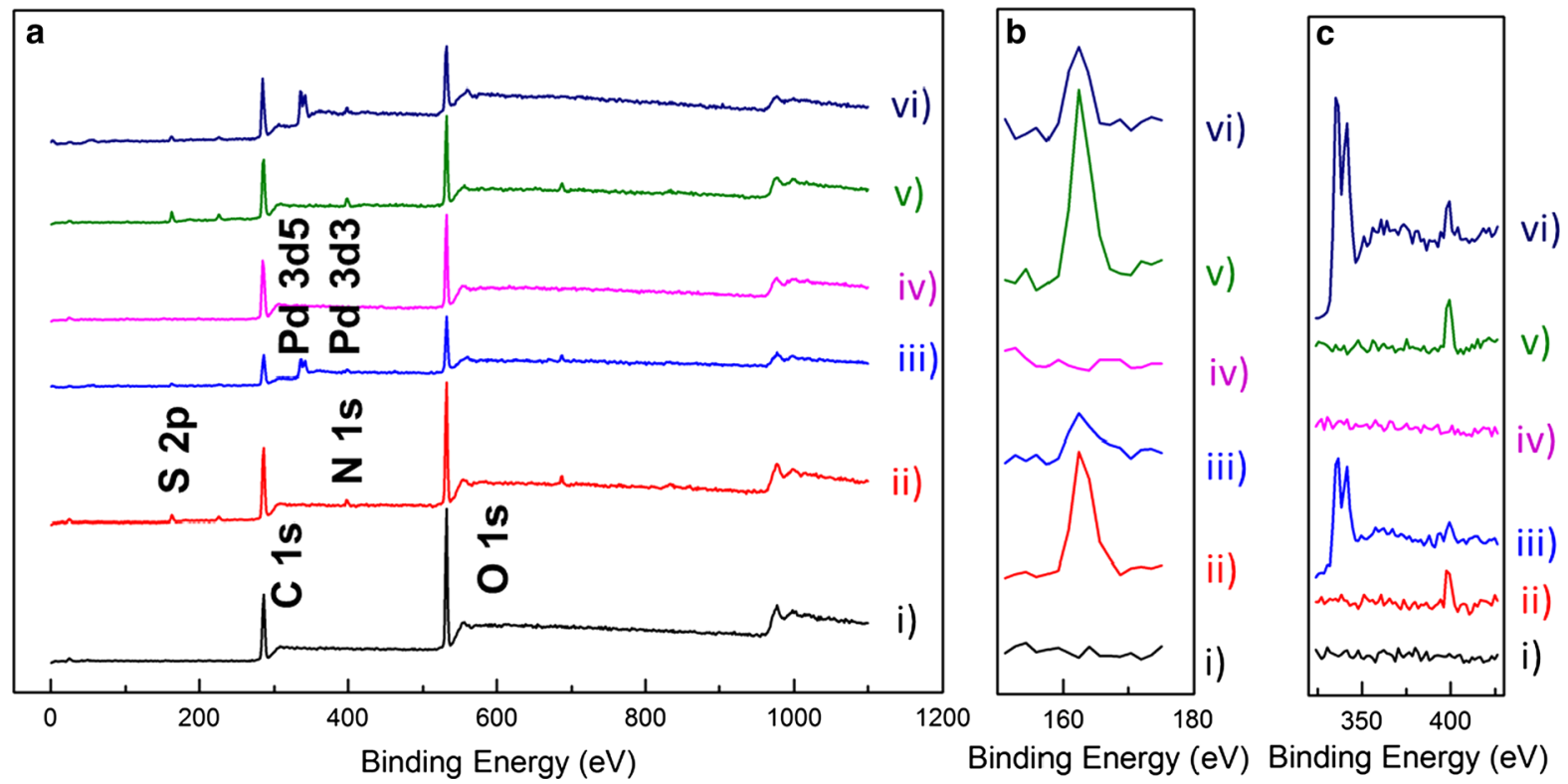

Fig. 6 XPS wide scans of a 0-1100 eV, b 150-180 eV and c 345-405 eV for i) 2D-DAC, ii) 2D-DAC-LC, iii) 2D-DAC-LC-Pd, iv) 13D-DAC, v) 13D-DAC-LC and vi) 13D-DAC-LC-Pd

DAC. Interestingly, after the modification with L-cysteine, the SSA increases significantly, to $141 \mathrm{~m}^{2} / \mathrm{g}$ for $2 \mathrm{D}-\mathrm{DAC}-\mathrm{LC}$ and to $47 \mathrm{~m}^{2} / \mathrm{g}$ for $13 \mathrm{D}-$ DAC-LC, which is supported by the more porous texture observed by SEM (see Fig. 4). In Fig. 8b, the pore size distributions of the samples are shown. All of the samples have a pore size range primarily in the mesoporous region $(2-50 \mathrm{~nm})$, except for $2 \mathrm{D}$-DAC-
LC, which has a broader pore size distribution with a mode at $45 \mathrm{~nm}$.

X-ray diffraction (XRD) spectroscopy

XRD curves for Cladophora cellulose, 2D-DAC, 2DDAC-LC，2D-DAC-LC-Pd，13D-DAC，13D-DACLC and 13D-DAC-LC-Pd are given in Fig. 9. As 

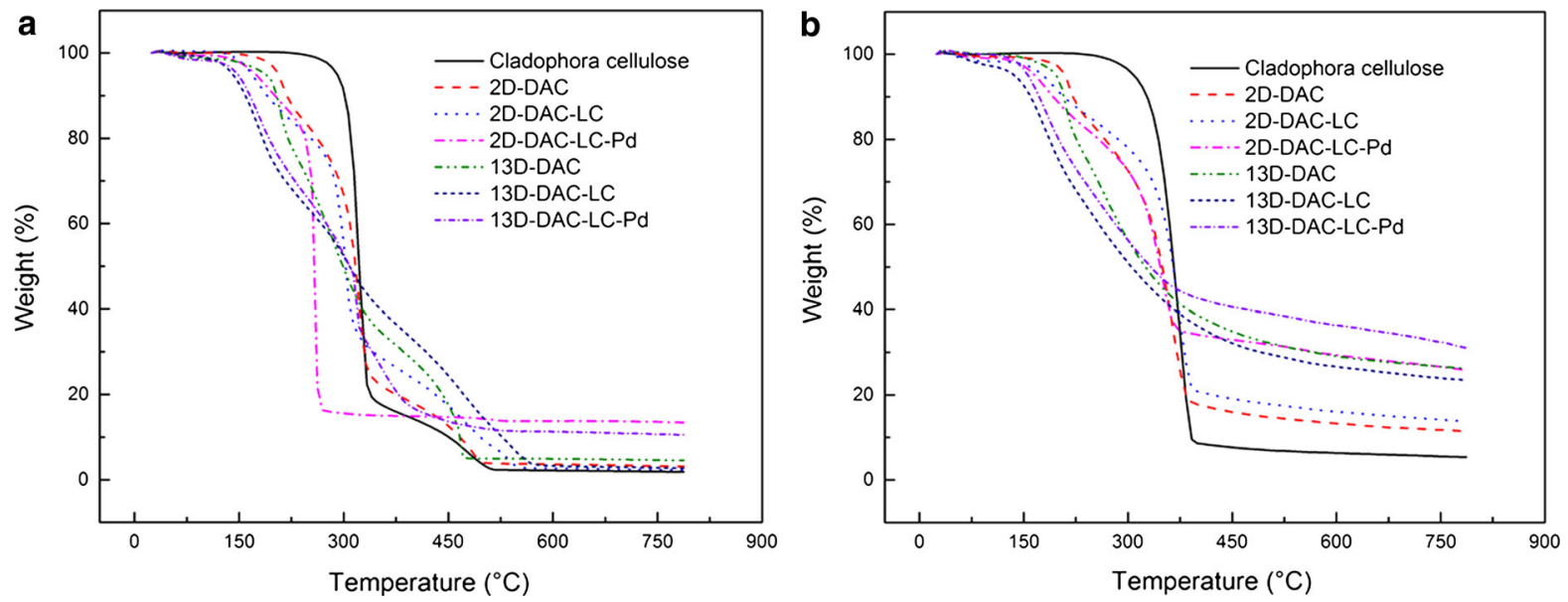

Fig. 7 Thermograms performed in a compressed air and b nitrogen for dried Cladophora nanocellulose, 2D-DAC, 2D-DAC-LC, 2DDAC-LC-Pd, 13D-DAC, 13D-DAC-LC and 13D-DAC-LC-Pd
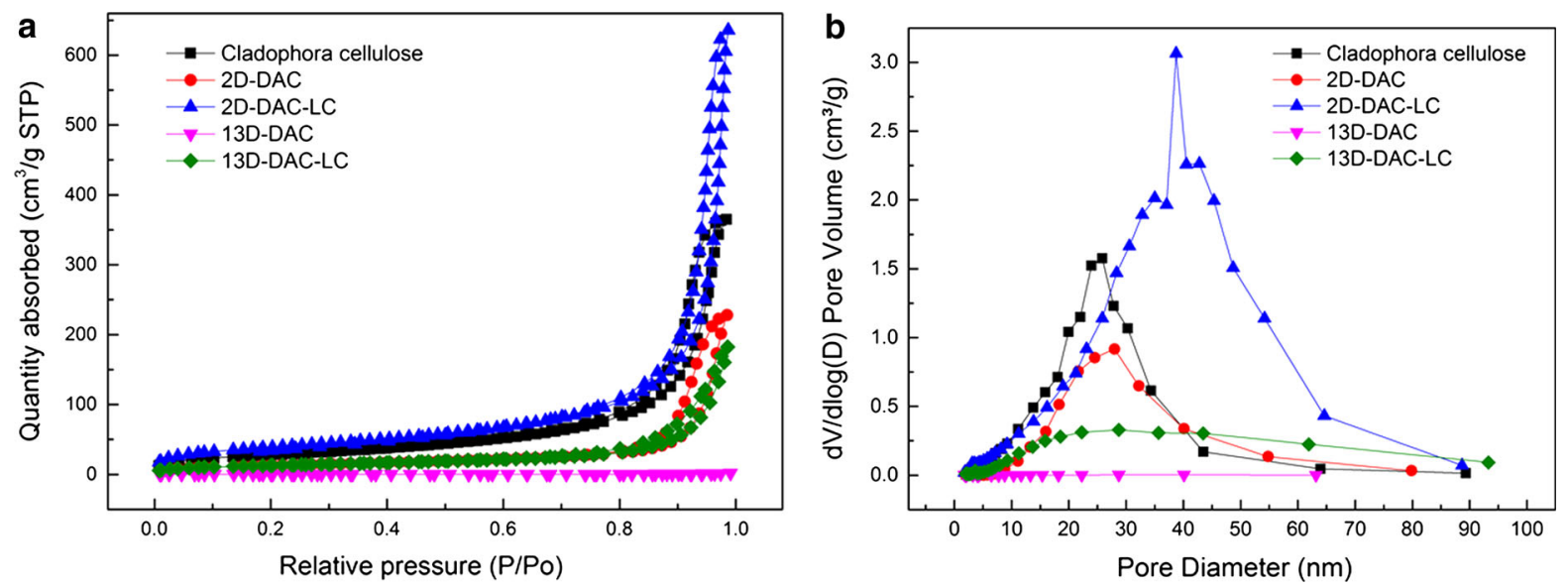

Fig. 8 a Nitrogen sorption isotherms $(T=77 \mathrm{~K})$ and $\mathbf{b}$ pore size distribution for cellulose and its derivatives

described in Segal's empirical equation (Segal et al. 1959), the intensity of 2-Theta at about $22.5^{\circ}$ and $18^{\circ}$ corresponds to crystalline and amorphous sections of cellulose, respectively. The intensity of peaks at about $22.5^{\circ}$ decreases for 2D-DAC, 2D-DAC-LC and 2DDAC-LC-Pd compared to unmodified cellulose, which indicates a decrease of crystallinity of the cellulose derivatives after chemical functionalization. Furthermore, the peaks at about $22.5^{\circ}$ disappear in the spectra of 13D-DAC, 13D-DAC-LC and 13D-DAC-LC-Pd, which suggest that these samples become completely amorphous.

\section{Conclusions}

The novel adsorption materials described herein provide a green and efficient alternative to frequently used adsorbents based on a petrol feedstock. The use of environmentally benign and renewable cellulose from the undesirable polluting green algae Cladophora, functionalized with the natural amino acid cysteine, constitutes a green approach to enable recovery of the transition metal palladium. The results from the adsorption study indicate that the adsorption of palladium ions is mainly governed by chemisorption. The 


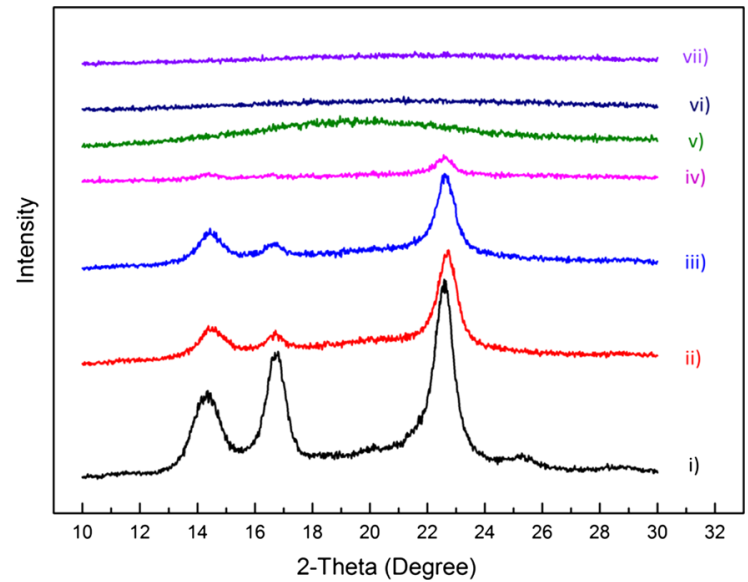

Fig. 9 XRD curves for $i$ ) Cladophora nanocellulose, ii) 2DDAC, iii) 2D-DAC-LC, iv) 2D-DAC-LC-Pd, v) 13D-DAC, vi) 13D-DAC-LC and vii) 13D-DAC-LC-Pd

adsorbent has a high adsorption capacity and enables fast adsorption of palladium ions from solution. With our process, materials suitable for both filters (fibrous materials) and for column matrixes (spherical beads) can be obtained. The adsorption materials were extensively characterized using several different methods e.g. FTIR, SEM, XRD, XPS, TGA, elemental analysis and gas adsorption.

Acknowledgments Ollie and Elof Ericssońs Foundation as well as the Bo Rydin Foundation are gratefully acknowledged for their financial support. Changqing Ruan thanks the China Scholarship Council (CSC) for financial support.

\section{References}

Anpilogova GR, Bondareva SO, Khisamutdinov RA, Murinov YI (2014) Fatty imidazolines: a novel extractant for the recovery of palladium(ii) from hydrochloric acid solutions. Solv Extr Ion Exch 32:206-220. doi:10.1080/07366299. 2013.838498

Awual MR, Yaita T, El-Safty SA, Shiwaku H, Okamoto Y, Suzuki S (2013) Investigation of palladium(II) detection and recovery using ligand modified conjugate adsorbent. Chem Eng J 222:172-179. doi:10.1016/j.cej.2013.02.058

Barakat MA, Mahmoud MHH, Mahrous YS (2006) Recovery and separation of palladium from spent catalyst. Appl Catal A 301:182-186. doi:10.1016/j.apcata.2005.11.028

Brunauer S, Emmett PH, Teller E (1938) Adsorption of gases in multimolecular layers. J Am Chem Soc 60:309-319. doi:10.1021/ja01269a023

Bruneel D, Schacht E (1993) Chemical modification of pullulan: 1. Periodate oxidation. Polymer 34:2628-2632. doi:10. 1016/0032-3861(93)90600-F
Carlsson DO, Lindh J, Nyholm L, Strømme M, Mihranyan A (2014) Cooxidant-free TEMPO-mediated oxidation of highly crystalline nanocellulose in water. Rsc Advances 4:52289-52298. doi:10.1039/c4ra11182f

Carlsson DO, Lindh J, Strømme M, Mihranyan A (2015) Susceptibility of $\mathrm{I}_{\alpha^{-}}$and $\mathrm{I}_{\beta}$-dominated cellulose to tempomediated oxidation. Biomacromolecules 16:1643-1649. doi:10.1021/acs.biomac.5b00274

Casu B, Naggi A, Torri G, Allegra G, Meille SV, Cosani A, Terbojevich M (1985) Stereoregular acyclic polyalcohols and polyacetates from cellulose and amylose. Macromolecules 18:2762-2767. doi:10.1021/ma00154a068

Cosenza VA, Navarro DA, Stortz CA (2011) Usage of alphapicoline borane for the reductive amination of carbohydrates. Org Chem Argent 2011:182-194. doi:10.3998/ark. 5550190.0012 .716

Freundlich HMA (1906) Concerning adsorption in solutions. J Phys Chem 57:385-470

Fujiwara K, Ramesh A, Maki T, Hasegawa H, Ueda K (2007) Adsorption of platinum (IV), palladium (II) and gold (III) from aqueous solutions onto L-lysine modified crosslinked chitosan resin. J Hazard Mater 146:39-50. doi:10.1016/j. jhazmat.2006.11.049

Gericke M, Trygg J, Fardim P (2013) Functional cellulose beads: preparation, characterization, and applications. Chem Rev 113:4812-4836. doi:10.1021/cr300242j

Gupta VK, Jain CK, Ali I, Sharma M, Saini VK (2003) Removal of cadmium and nickel from wastewater using bagasse fly ash-a sugar industry waste. W Res 37:4038-4044. doi:10. 1016/S0043-1354(03)00292-6

Gupta VK, Mittal A, Gajbe V, Mittal J (2006) Removal and recovery of the hazardous azo dye acid orange 7 through adsorption over waste materials: bottom ash and de-oiled soya. Ind Eng Chem Res 45:1446-1453. doi:10.1021/ ie051111f

Jermakowicz-Bartkowiak D, Kolarz BN, Serwin A (2005) Sorption of precious metals from acid solutions by functionalised vinylbenzyl chloride-acrylonitryle-divinylbenzene copolymers bearing amino and guanidine ligands. React Funct Polym 65:135-142. doi:10.1016/j. reactfunctpolym.2004.11.010

Kitkulnumchai Y, Ajavakom A, Sukwattanasinitt M (2008) Treatment of oxidized cellulose fabric with chitosan and its surface activity towards anionic reactive dyes. Cellulose 15:599-608. doi:10.1007/s10570-008-9214-8

Klemm D, Heublein B, Fink HP, Bohn A (2005) Cellulose: fascinating biopolymer and sustainable raw material. Angew Chem-Int Edit 44:3358-3393. doi:10.1002/anie. 200460587

Lagergren S (1898) Kungliga Svenska Vetenskapsakademiens, vol 24. Vetensk, Handl

Langmuir I (1918) The adsorption of gases on plane surfaces of glass, mica and platinum. J Am Chem Soc 40:1361-1403. doi:10.1021/ja02242a004

Li GQ, Konishi S, Saito K, Sugo T (1994) High collection rate of Pd in hydrochloric-acid medium using chelating microporous membrane. J Membr Sci 95:63-69. doi:10.1016/ 0376-7388(94)85029-1

Lim JS, Kim SM, Lee SY, Stach EA, Culver JN, Harris MT (2011) Surface functionalized silica as a toolkit for studying aqueous phase palladium adsorption and 
mineralization on thiol moiety in the absence of external reducing agents. J Coll Interf Sci 356:31-36. doi:10.1016/j. jcis.2010.12.064

Lindh J, Carlsson DO, Strømme M, Mihranyan A (2014) Convenient one-pot formation of 2,3-dialdehyde cellulose beads via periodate oxidation of cellulose in water. Biomacromolecules 15:1928-1932. doi:10.1021/bm5002944

Lindh J, Ruan C, Strømme M, Mihranyan A (2016) Preparation of porous cellulose beads via introduction of diamine spacers. Langmuir. doi:10.1021/acs.langmuir.6b01288

Liu K, Yen W, Shibayama A, Fujita T (2003) Selective leaching of platinum and palladium by chloride solution. In: Proceedings of the TMS Fall Extraction and Processing Conference, pp 1617-1628

Maekawa E, Koshijima T (1984) Properties of 2,3-dicarboxy cellulose combined with various metallic ions. J Appl Polym Sci 29:2289-2297. doi:10.1002/app.1984.070290705

Metreveli G, Wagberg L, Emmoth E, Belak S, Stromme M, Mihranyan A (2014) A size-exclusion nanocellulose filter paper for virus removal. Adv Healthc Mater 3:1546-1550. doi:10.1002/adhm.201300641

Mihranyan A (2011) Cellulose from Cladophorales green algae: from environmental problem to high-tech composite materials. J Appl Polym Sci 119:2449-2460. doi:10.1002/ app.32959

Moulder JF, Stickle WF, Sobol PE, Bomben KD (1992) Handbook of X-ray photoelectron spectroscopy. PerkinElmer Corp, Palo Alto

Nada A, Hassan ML (2006) Ion exchange properties of carboxylated bagasse. J Appl Polym Sci 102:1399-1404. doi:10.1002/app.24255

Nolan EM, Lippard SJ (2008) Tools and tactics for the optical detection of mercuric ion. Chem Rev 108:3443-3480. doi:10.1021/cr068000q

Nyholm L, Nyström G, Mihranyan A, Strømme M (2011) Toward flexible polymer and paper-based energy storage devices. Adv Mater 23:3751-3769. doi:10.1002/adma. 201004134

Paiva AP, Carvalho GI, Costa MC, da Costa AMR, Nogueira C (2014) Recovery of platinum and palladium from chloride solutions by a thiodiglycolamide derivative. Solv Extr Ion Exch 32:78-94. doi:10.1080/07366299.2013.810969

Panchompoo J, Aldous L, Compton RG (2011) Irreversible uptake of palladium from aqueous systems using L-cysteine methyl esterphysisorbed on carbon black. J Mater Chem 21:9513-9522. doi:10.1039/C0JM04493H

Products CfPM (17th December, 2002) European agency for the evaluation of medicinal products. Evaluation of Medicines for Human Use, London, UK

Qishu Q, Qian G, Zuli G, Yuqi S, Chengyin W, Xiaoya H (2012) Efficient removal of heavy metal from aqueous solution by sulfonic acid functionalized nonporous silica microspheres. Coll Surf Physicochem Eng Asp 415:41-46. doi:10.1016/j.colsurfa.2012.08.059

Razaq A, Nyholm L, Sjödin M, Strømme M, Mihranyan A (2012) Paper-based energy-storage devices comprising carbon fiber-reinforced polypyrrole-Cladophora nanocellulose composite electrodes. Adv Energy Mater 2:445-454. doi:10. 1002/aenm.201100713

Rowen JW, Forziati FH, Reeves RE (1951) Spectrophotometric evidence for the absence of free aldehyde groups in periodate-oxidized cellulose. J Am Chem Soc 73:4484-4487. doi: $10.1021 / \mathrm{ja} 01153 \mathrm{a} 535$

Ruhaak LR, Steenvoorden E, Koeleman CAM, Deelder AM, Wuhrer M (2010) 2-Picoline-borane: a non-toxic reducing agent for oligosaccharide labeling by reductive amination. Proteomics 10:2330-2336. doi:10.1002/pmic.200900804

Sabzalian Z, Alam MN, van de Ven TGM (2014) Hydrophobization and characterization of internally crosslink-reinforced cellulose fibers. Cellulose 21:1381-1393. doi:10. 1007/s10570-014-0178-6

Sağ Y (2001) Biosorption of heavy metals by fungal biomass and modeling of fungal biosorption: a review. Sep Purif Rev 30:1-48. doi:10.1081/SPM-100102984

Sato S, Sakamoto T, Miyazawa E, Kikugawa Y (2004) One-pot reductive amination of aldehydes and ketones with alphapicoline-borane in methanol, in water, and in neat conditions. Tetrahedron 60:7899-7906. doi:10.1016/j.tet.2004. 06.045

Segal L, Creely J, Martin A, Conrad C (1959) An empirical method for estimating the degree of crystallinity of native cellulose using the X-ray diffractometer. Text Res J 29:786-794

Sharma S, Rajesh N (2014) 2-Mercaptobenzothiazole impregnated cellulose prepared by ultrasonication for the effective adsorption of precious metal palladium. Chem Eng J 241:112-121. doi:10.1016/j.cej.2013.12.002

Sparks DL (ed) (1986) Kinetics of reactions in pure and in mixed systems. In: Soil physical chemistry. CRC Press, Boca Raton, pp 83-145

Spedding H (1960) Infrared spectra of periodate-oxidised cellulose. J Chem Soc 73:3147-3152. doi:10.1039/ JR9600003147

Su SX, Nutiu R, Filipe CDM, Li YF, Pelton R (2007) Adsorption and covalent coupling of ATP-binding DNA aptamers onto cellulose. Langmuir 23:1300-1302. doi:10.1021/la060961c

Takaichi S, Hiraoki R, Inamochi T, Isogai A (2014) One-step preparation of 2,3,6-tricarboxy cellulose. Carbohydr Polym 110:499-504. doi:10.1016/j.carbpol.2014.03.085

Venkatesan KA, Selvan BR, Antony MP, Srinivasan TG, Rao PRV (2005) Extraction of palladium from nitric acid medium by commercial resins with phosphinic acid, methylene thiol and isothiouronium moieties attached to polystyrene-divinylbenzene. J Radioanal Nucl Chem 266:431-440. doi:10.1007/s10967-005-0928-x

Vincent T, Guibal E, Chiarizia R (2007) Palladium recovery by reactive precipitation using a cyanex 301-based stable emulsion. Sep Sci Technol 42:3517-3536. doi:10. 1080/01496390701626735

Wasikiewicz JM, Mitomo H, Seko N, Tamada M, Yoshii F (2007) Platinum and palladium ions adsorption at the trace amounts by radiation crosslinked carboxymethylchitin and carboxymethylchitosan hydrogels. J Appl Polym Sci 104:4015-4023. doi:10.1002/app.26034 